\title{
Generalized assisted inflation
}

\author{
E. J. Copeland \\ Centre for Theoretical Physics, University of Sussex, Falmer, Brighton BN1 9QJ, United Kingdom \\ Anupam Mazumdar \\ Astrophysics Group, Blackett Laboratory, Imperial College, London SW7 2BZ, United Kingdom \\ N. J. Nunes \\ Centre for Theoretical Physics, University of Sussex, Falmer, Brighton BN1 9QJ, United Kingdom
}

(Received 22 April 1999; published 15 September 1999)

\begin{abstract}
We obtain a new class of exact cosmological solutions for multiscalar fields with exponential potentials. We generalize the assisted inflation solutions previously obtained, and demonstrate how they are modified when there exist cross couplings between the fields, such as occur in supergravity inspired cosmological models. [S0556-2821(99)04216-2]
\end{abstract}

PACS number(s): 98.80.Cq

\section{INTRODUCTION}

Scalar field theory has become the generic playground for building cosmological models related to particle physics, in particular for obtaining inflationary cosmologies. One such class of models involves exponential potentials $\left[\exp \left(\sqrt{16 \pi / p m_{\mathrm{Pl}}^{2}} \phi\right)\right]$, which lead to power law inflation, $a$ $\propto t^{p}$, with $p>1$, for sufficiently flat potentials [1-3]. A number of related features have also been discovered for such potentials: in a universe containing a perfect fluid and such a scalar field, then for a wide range of parameters the scalar field "mimics" the perfect fluid, adopting its equation of state [4-6] and leading to attractor scaling solutions at late time [7]. These solutions offer a plausible mechanism for stabilizing the dilaton field in models of gaugino condensation arising in supersymmetry breaking [8].

It is generally assumed that even if there are many scalar fields present, only one of them will dominate the dynamics and roll down the potential slowly. However, recently Liddle, Mazumdar, and Schunck (LMS) [9] have demonstrated in a particular example that multiple scalar fields, each with an exponential potential, can lead to inflationary solutions, even if the individual field potentials are too steep for inflation. There exists a cumulative effect of all the fields that can give rise to inflationary behavior-a result they termed "assisted inflation." Malik and Wands have demonstrated that the associated attractor solution could be identified through a rotation in field space, with a hybrid model where the vacuum energy had an exponential dependence upon the dilaton field [10]. Multiple exponential potentials do arise in modern Kaluza-Klein theories. Indeed, they are a natural outcome of the compactification of higher dimensional theories down to $3+1$ dimensions. With this in mind it is worth investigating such potentials in a bit more detail. Indeed, Kanti and Olive have recently proposed a possible realization of assisted inflation based on the compactification of a five-dimensional Kaluza-Klein model [11]. It also raises the question, could inflation arise out of the 11-dimensional supergravity models compactified on squashed seven spheres for example? Such models have been investigated by a num- ber of authors as low-energy cosmologies from string or $\mathrm{M}$ theory [12-15]. Most "realistic' models of dimensional reduction lead to steep potentials, which generally do not lead to inflation. In this paper we consider a more general class of exponential potentials, which can include those generally found in supergravity compactifications, and obtain exact cosmological solutions for them. In particular we demonstrate how difficult it is to obtain assisted inflation when there exist cross couplings between the scalar fields in the potential, a result also discussed in [14] and [11]. We first recall the model discussed by LMS, before generalizing their potential to exponentials involving cross-coupling terms and demonstrating that the attractor behavior of the scalar fields still exists, leading to scaling solutions for the generalized potential. We then turn our attention to the case of potentials involving multiple exponential terms containing the same scalar fields, and relate the solutions to those arising in supergravity models.

\section{THE DYNAMICS OF ASSISTED INFLATION}

Liddle et al. [9], considered $n$ scalar fields, $\phi_{i}, i$ $=1, \ldots n$, each with exponential potentials decoupled from each other:

$$
V_{i}\left(\phi_{i}\right)=V_{0} \exp \left(\alpha_{i} \phi_{i}\right)
$$

where $\alpha_{i}$ is the slope of the individual field with dimensions of the inverse Planck mass. Although the fields are not directly coupled through the potential, they are coupled through the Friedmann equation, which implies that the combined role of the fields affect the expansion rate of the universe:

$$
\begin{gathered}
H^{2}=\frac{8 \pi}{3 m_{\mathrm{Pl}}^{2}} \sum_{i=1}^{n}\left[V_{i}\left(\phi_{i}\right)+\frac{1}{2} \dot{\phi}_{i}^{2}\right], \\
\ddot{\phi}_{i}=-3 H \dot{\phi}_{i}-\frac{d V_{i}\left(\phi_{i}\right)}{d \phi_{i}},
\end{gathered}
$$


where $H=\dot{a} / a$ is Hubble's constant and $a$ is the scale factor of the flat Friedmann-Robertson-Walker (FRW) universe. The solution to this is the modified power law [9]

$$
a(t) \propto t^{p},
$$

where $p$ is given by

$$
p=\frac{16 \pi}{m_{\mathrm{Pl}}^{2}} \sum_{i=1}^{n} \frac{1}{\alpha_{i}^{2}}
$$

Inflationary solutions exist provided $p>1$, hence even if each of the $\alpha_{i}$ 's are too steep to individually satisfy the condition for inflation, as long as $n$ is large enough, the inequality $p>1$ can be satisfied. These solutions, and the inflationary ones we shall present below are eternal, they do not possess an exit from the inflationary epoch. Realistic models would of course have to possess such an exit in order to enter the radiation- and matter-dominated epochs of our universe. The particular example of Eq. (5) suggests that it is worth investigating whether or not such assisted inflation exists with more general exponential potentials. An alternative approach with interesting results has been adopted in [11], where they have applied the assistance method to the case of polynomial scalar potentials.

\section{Exponential potentials with coupled scalar fields}

To begin with we consider the most natural generalization of the single field exponential, namely the case of two coupled fields:

$$
V(\phi, \psi)=V_{0} e^{\alpha \phi+\beta \psi},
$$

where $\alpha$ and $\beta$ are the slopes for the fields $\phi$ and $\psi$. We see from Eq. (2) that dimensionally, the right-hand side should decrease as $t^{-2}$, because $H^{2} \propto t^{-2} \propto V_{i}\left(\phi_{i}\right)$. We further assume that, for our potential Eq. (6),

$$
e^{\alpha \phi}=\frac{k_{\alpha}}{t^{c}}, \quad e^{\beta \psi}=\frac{k_{\beta}}{t^{2-c}},
$$

where $k_{\alpha}, k_{\beta}$ are dimensional constants and $c$ is a dimensionless constant. Substituting the power-law solution Eq. (4) into Eq. (2) we obtain

$$
p^{2}=H^{2} t^{2}=\frac{8 \pi}{3 m_{\mathrm{Pl}}^{2}}\left[V_{0} k_{\alpha} k_{\beta}+\frac{1}{2}\left(\frac{c}{\alpha}\right)^{2}+\frac{1}{2}\left(\frac{2-c}{\beta}\right)^{2}\right],
$$

which when coupled with Eq. (3) for the $\phi$ and $\psi$ fields leads to

$$
V_{0} k_{\alpha} k_{\beta}=\frac{(3 p-1) c}{\alpha^{2}}, \quad V_{0} k_{\alpha} k_{\beta}=\frac{(3 p-1)(2-c)}{\beta^{2}},
$$

hence

$$
V_{0} k_{\alpha} k_{\beta}=\frac{2(3 p-1)}{\alpha^{2}+\beta^{2}} .
$$

Using Eqs. (9) and (10) in Eq. (8), we obtain a simple scaling solution between the two fields:

$$
p=\frac{16 \pi}{m_{\mathrm{Pl}}^{2}} \frac{1}{\alpha^{2}+\beta^{2}}
$$

$$
\left(\frac{\dot{\phi}}{\dot{\psi}}\right)^{2}=\left(\frac{\alpha}{\beta}\right)^{2}
$$

An important problematic feature for inflationary solutions confronts us immediately in Eq. (11), namely the coupling between the two fields reduces the rate of expansion of the universe, a point also made in $[14,11]$. We will return to this point again later. An alternative method which would also lead to Eq. (11) is described in [10] in terms of field rotations, which results in the introduction of two orthogonal fields, one of which is massless and the other posseses an exponential potential.

Having demonstrated that it is possible to obtain a scaling solution without using slow roll approximations, we now generalize this simple case to include an arbitrary number of fields and exponential terms making up the overall potential.

\section{General exponential potentials with coupled scalar fields}

We now consider a potential where we have multiple scalar fields but their corresponding exponential potentials can contain arbitrary combinations of the fields with different slopes. The potential we will consider is

$$
V=\sum_{s=1}^{n} V_{s}=V_{0} \sum_{s=1}^{n} \exp \left(\sum_{j=1}^{m_{s}} \alpha_{s j} \phi_{s j}\right),
$$

with the corresponding Friedmann equation

$$
H^{2}=\frac{8 \pi}{3 m_{\mathrm{Pl}}^{2}}\left[V+\sum_{s=1}^{n} \sum_{j=1}^{m_{s}} \frac{1}{2} \dot{\phi}_{s j}^{2}\right],
$$

where, from now on $q, r, s$ stands for index terms in the potential and $i, j, k, l$ stands for field indexes, hence, $\phi_{s j}$ stands for the $j$ th field in the $s$ th potential term. In other words, there are a total of $\sum_{s=1}^{n} m_{s}$ fields distributed in groups of $m_{s}$ through the terms of the potential. We obtain the solution to this problem by generalizing the assumption Eq. (7). There exists an attractor region with a power-law solution, which from Eq. (14), dimensionally satisfies $H^{2}$ $\propto t^{-2} \propto V_{i}$. Hence, we write

$$
\begin{gathered}
e^{\alpha_{s j} \phi_{s j}}=\frac{k_{s j}}{t^{c_{s j}}}, \\
\sum_{j=1}^{m_{s}} c_{s j}=2,
\end{gathered}
$$

where $k_{s j}$ are dimensional and $c_{s j}$ are dimensionless constants, respectively. Equation (15), coupled with the equations of motion 


$$
\ddot{\phi}_{s j}+3 H \dot{\phi}_{s j}+\frac{\partial V}{\partial \phi_{s j}}=0,
$$

result in

$$
(3 p-1) c_{s j}=\alpha_{s j}^{2} V_{0} \prod_{k=1}^{m_{s}} k_{s k},
$$

from which we find, using Eqs. (16) and (17):

$$
\begin{gathered}
V_{0} \prod_{k=1}^{m_{s}} k_{s k}=\frac{2(3 p-1)}{\sum_{j=1}^{m_{s}} \alpha_{s j}^{2}}, \\
\left(\frac{c_{s j}}{\alpha_{s j}}\right)^{2}=\frac{4 \alpha_{s j}^{2}}{\left(\sum_{k=1}^{m_{s}} \alpha_{s k}^{2}\right)^{2}} .
\end{gathered}
$$

When substituted into Eq. (14) this leads to a key result, the generalization of the original assisted inflation result given by Eq. (5):

$$
p=\frac{16 \pi}{m_{\mathrm{Pl}}^{2}} \sum_{s=1}^{n} \frac{1}{\sum_{j=1}^{m_{s}} \alpha_{s j}^{2}}
$$

We also note that the generalization of the scaling solution found in Eq. (11), quickly follows for the case of any two scalar fields, $\phi_{s j}$ and $\phi_{q l}$ :

$$
\left(\frac{\dot{\phi}_{s j}}{\dot{\phi}_{q l}}\right)^{2}=\left(\frac{\alpha_{s j}}{\alpha_{q l}}\right)^{2}\left(\frac{\sum_{i=1}^{m_{q}} \alpha_{q i}^{2}}{\sum_{k=1}^{m_{s}} \alpha_{s k}^{2}}\right)^{2} .
$$

It is directly evident that Eqs. (20) and (21) reduce to Eq. (5) for the example of $n$ exponential terms each containing just one field, and Eq. (11) for the case of one exponential term but containing two fields. We again see the inhibiting affect that multiplicative coupling of the fields (i.e., $m_{s}>1$ ) has for obtaining inflationary solutions. However, in this case, as with the original version of assisted inflation, this can be compensated for if there are enough exponential terms present in the potential (i.e., if $n$ is large enough) [9].

There is another feature of the potentials we have been discussing so far that makes them rather unphysical in general. We have been demanding that any two fields present cannot be the same (i.e., $\phi_{s j} \neq \phi_{q l}$ ). In other words they can only appear once in the full potential. Nearly all realistic models which emerge from compactifications arising in supergravity models have the same field appearing in at least two separate exponential terms. In the following section, we turn our attention to this case.

\section{EXPONENTIAL POTENTIALS INSPIRED BY SUPERGRAVITY MODELS}

To set the scene, we generalize Eq. (6) to the case where the scalar field potential takes the following form:

$$
V\left(\phi_{1}, \phi_{2}\right)=z_{1} e^{\alpha_{11} \phi_{1}+\alpha_{12} \phi_{2}}+z_{2} e^{\alpha_{21} \phi_{1}+\alpha_{22} \phi_{2}},
$$

where, $\alpha_{s j}$, are dimensional constants which can take any real value and $z_{s}>0$. The occurrence of such forms of the potential are quite common in dimensionally reduced supergravity models $[13,14]$. Remarkably we can solve this system to obtain scaling solutions in a manner analogous to those already presented in Eqs. (7)-(11) obtaining the unique late time scaling solution for the fields $\phi_{1}$ and $\phi_{2}$,

$$
p=\frac{16 \pi}{m_{\mathrm{Pl}}^{2}}\left[\frac{\left(\alpha_{21}-\alpha_{11}\right)^{2}+\left(\alpha_{22}-\alpha_{12}\right)^{2}}{\left(\alpha_{11} \alpha_{22}-\alpha_{21} \alpha_{12}\right)^{2}}\right] .
$$

This simple result reduces to the particular cases we have already investigated when the appropriate limits are taken. For example, when $\alpha_{21}=\alpha_{12}=0$, we reproduce the result Eq. (5). The equivalent of the assisted inflation result Eq. (11) follows by setting $\alpha_{11} \alpha_{21}+\alpha_{12} \alpha_{22}=0$, in which case we find

$$
p=\frac{16 \pi}{m_{\mathrm{Pl}}^{2}}\left[\frac{1}{\alpha_{11}^{2}+\alpha_{12}^{2}}+\frac{1}{\alpha_{21}^{2}+\alpha_{22}^{2}}\right] .
$$

We shall now generalize the potential to $n$ such exponential potentials and $m$ combinations of linear fields in the exponent [explicitly calculating for the simple case of 2 terms $\times 2$ fields of Eq. (22)]. The generalized Eq. (22) is then

$$
V=\sum_{s=1}^{n} z_{s} \exp \left(\sum_{j=1}^{m} \alpha_{s j} \phi_{j}\right) .
$$

Of course, we are allowing here for the possibility that $\alpha_{s j}$ $=0$ for some combination of $s j$. We assume that for late times the fields have an attractor solution, given by

$$
z_{s} \exp \left(\sum_{j=1}^{m} \alpha_{s j} \phi_{j}\right)=\frac{k_{s}}{t^{2}},
$$

and, following Eqs. (15) and (16) we write

$$
\phi_{j}=a_{j}-\frac{c_{s j}}{\alpha_{s j}} \ln t
$$

where, $a_{j}$ is a constant depending on the initial conditions and $\sum_{j=1}^{m} c_{s j}=2, s=1, \ldots, n$. Substituting Eq. (27) into the equation of motion Eq. (3), we obtain the constraint equation for the $j$ th field, which follows from assuming the existence of a power-law solution

$$
(3 p-1) \frac{c_{s j}}{\alpha_{s j}}=\sum_{q=1}^{n} \alpha_{q j} k_{q}
$$

Again, using $\sum_{j=1}^{m} c_{i j}=2$ we obtain 


$$
\sum_{j=1}^{m} \alpha_{s j}\left(\sum_{q=1}^{n} \alpha_{q j} k_{q}\right)=2(3 p-1)
$$

which is equivalent to writing

$$
\sum_{q=1}^{n} A_{s q} k_{q}=2(3 p-1)
$$

where

$$
A_{s q}=\sum_{j=1}^{m} \alpha_{s j} \alpha_{q j}
$$

Since $s$ is a free index, we have a set of $n$ equations that can be written as

$$
A \mathbf{k}=2(3 p-1),
$$

where $A$ is the $n \times n$ matrix with elements $A_{s q}$ and $\mathbf{k}$ $=\left(k_{1}, \ldots, k_{n}\right)$ a column vector. For the $2 \times 2$ case of Eq. (22) we obtain

$$
A=\left(\begin{array}{cc}
\alpha_{11}^{2}+\alpha_{12}^{2} & \alpha_{11} \alpha_{21}+\alpha_{12} \alpha_{22} \\
\alpha_{21} \alpha_{11}+\alpha_{22} \alpha_{12} & \alpha_{21}^{2}+\alpha_{22}^{2}
\end{array}\right) .
$$

The solution to this system is

$$
\mathbf{k}=A^{-1} 2(3 p-1),
$$

with

$$
A^{-1}=\frac{A_{\mathrm{COF}}^{T}}{\operatorname{det} A},
$$

where $A_{\mathrm{COF}}^{T}$ is the transpose of the cofactor matrix of $A$. To simplify notation we will write $B \equiv A_{\mathrm{COF}}^{T}$ and the sum of the elements in row $s$ of $B$ as $B^{s} \equiv \sum_{q=1}^{n} B_{s q}$, hence, each $k_{s}$ is

$$
k_{s}=\frac{2(3 p-1)}{\operatorname{det} A} B^{s} .
$$

For the $2 \times 2$ case this yields

$$
\begin{gathered}
B=\left(\begin{array}{cc}
\alpha_{21}^{2}+\alpha_{22}^{2} & -\alpha_{11} \alpha_{21}-\alpha_{12} \alpha_{22} \\
-\alpha_{21} \alpha_{11}-\alpha_{22} \alpha_{12} & \alpha_{11}^{2}+\alpha_{12}^{2}
\end{array}\right), \\
k_{1}=2(3 p-1) \frac{\alpha_{21}^{2}+\alpha_{22}^{2}-\alpha_{11} \alpha_{21}-\alpha_{12} \alpha_{22}}{\left(\alpha_{11} \alpha_{22}-\alpha_{12} \alpha_{21}\right)^{2}}, \\
k_{2}=2(3 p-1) \frac{-\alpha_{21} \alpha_{11}-\alpha_{22} \alpha_{12}+\alpha_{11}^{2}+\alpha_{12}^{2}}{\left(\alpha_{11} \alpha_{22}-\alpha_{12} \alpha_{21}\right)^{2}} .
\end{gathered}
$$

From Eqs. (27) and (28) the late time ratio between the kinetic terms of two different fields becomes

$$
\left(\frac{\dot{\phi}_{j}}{\dot{\phi}_{l}}\right)^{2}=\left(\frac{\sum_{q=1}^{n} \alpha_{q j} B^{q}}{\sum_{r=1}^{n} \alpha_{r l} B^{r}}\right)^{2} .
$$

Substitution of Eqs. (36) and (28) into the Friedmann equation yields

$$
\begin{aligned}
p^{2} & =\frac{8 \pi}{3 m_{\mathrm{Pl}}^{2}}\left[\sum_{s=1}^{n} k_{s}+\frac{1}{2} \sum_{j=1}^{m}\left(\frac{c_{s j}}{\alpha_{s j}}\right)^{2}\right] \\
& =\frac{8 \pi}{3 m_{\mathrm{Pl}}^{2}}\left[2(3 p-1) \sum_{s=1}^{n} \frac{B^{s}}{\operatorname{det} A}+2 \sum_{j=1}^{m}\left(\frac{\sum_{q=1}^{n} \alpha_{q j} B^{q}}{\operatorname{det} A}\right)^{2}\right] .
\end{aligned}
$$

After some algebra, we obtain the simple result for $p$ as the ratio between the sum of all the elements in the cofactor matrix of $A$ and its determinant

$$
p=\frac{16 \pi}{m_{\mathrm{Pl}}^{2}} \frac{\sum_{s}^{n} \sum_{q}^{n} B_{s q}}{\operatorname{det} A} .
$$

The reader should have no problem showing that for the 2 $\times 2$ case this reduces to Eq. (23).

It is instructive to rewrite Eq. (41) in another form. From Eqs. (30), (36), and (41),

$$
p=\frac{16 \pi}{m_{\mathrm{Pl}}^{2}} \sum_{s=1}^{n} \frac{1}{\sum_{q=1}^{n} A_{s q} k_{q} / k_{s}},
$$

and using Eq. (31) with $q=s$ and Eq. (36) we obtain

$$
p=\frac{16 \pi}{m_{\mathrm{Pl}}^{2}} \sum_{s=1}^{n} \frac{1}{\sum_{j=1}^{m} \alpha_{s j}^{2}+\sum_{q \neq s}^{n} A_{s q} B^{q} / B^{s}} .
$$

A number of points need to be made about Eq. (43). It is similar in form to Eq. (20), which should not be too surprising, the additional terms in the denominator arising from the fact that we have allowed for fields to appear more than once in the potential, hence leading effectively to "selfinteraction"-type contributions. Indeed if these terms were turned off we would reproduce the result in Eq. (20). In the $2 \times 2$ case, it is the constraint leading to Eq. (24). Due to the presence of these "self-interaction" terms, $p$ could increase above the value in Eq. (20) if there happened to be a combination of positive and negative slopes in Eq. (43).

An issue emerges when considering these more complicated potentials. For the two field, two term case of Eq. (22), if $\alpha_{11}>\alpha_{12}$ then, a necessary (but not sufficient) condition for the second term to be comparable to the first term at late times is, $\alpha_{21}<\alpha_{22}$. By comparable we mean that the potential terms reach a constant ratio. If this were not the case, 
then one of the two terms would quickly dominate the overall dynamics. One way to check if a combination of terms in a given potential will be comparable at late times is to use Eq. (36) to obtain the ratios $k_{s} / k_{q}$ for these terms. From Eq. (26) it follows that for consistency we require them to be positive, with $p>1 / 3$. In general, the surviving terms (i.e., those which remain comparable) will be the ones with the smallest slopes, corresponding to the largest values of $p$.

\section{CONCLUSIONS}

In this paper we have derived a new class of exact cosmological solutions involving exponential potentials. In doing so we have been able to generalize the assisted inflation solutions discussed by LMS [9] to the case of multiple exponential terms involving many fields. Such potentials are more likely to arise in realistic models of particle physics where individual fields will occur in a number of separate exponential terms, leading to cross couplings between the terms. In general, it transpires that it is more difficult to obtain assisted inflation in such models, the fields in any one exponential term tend to conspire to act against one another rather than assist each other, a result also noticed in $[14,11]$. This is the real reason why such models tend to fail to produce inflationary solutions in supergravity models compactified on squashed seven spheres [14]. We also investigated the case where a number of exponential terms contained the same scalar fields and demonstrated that a number of novel features emerged, including the possibility of increasing the rate of expansion when there exists a mixture of positive and negative slopes in the potential.

\section{ACKNOWLEDGMENTS}

We would like to thank Andrew Liddle, Karim Malik, David Wands, and Orfeu Bertolami for useful discussions. E.J.C. was supported by PPARC and is particularly grateful to David Wands for conversations on the nature of the generalized solutions. N.J.N. was supported by FCT (Portugal) under Contract No. PRAXIS XXI BD/15736/98 and A.M. was supported by the INLAKS and the ORS.
[1] F. Lucchin and S. Matarrese, Phys. Rev. D 32, 1316 (1985).

[2] J.J. Halliwell, Phys. Lett. B 185, 341 (1987).

[3] A.B. Burd and J.D. Barrow, Nucl. Phys. B308, 929 (1988).

[4] C. Wetterich, Nucl. Phys. B302, 668 (1988).

[5] D. Wands, E.J. Copeland, and A.R. Liddle, Ann. (N.Y.) Acad. Sci. 688, 647 (1993).

[6] P.G. Ferreira and M. Joyce, Phys. Rev. D 58, 023503 (1998).

[7] E.J. Copeland, A.R. Liddle, and D. Wands, Phys. Rev. D 57, 4686 (1998).

[8] T. Barreiro, B. de Carlos, and E.J. Copeland, Phys. Rev. D 58, 083513 (1998).
[9] A.R. Liddle, A. Mazumdar, and F.E. Schunck, Phys. Rev. D 58, 061301 (1998).

[10] K. Malik and D. Wands, Phys. Rev. D 59, 123501 (1999).

[11] P. Kanti and K.A. Olive, Phys. Rev. D 60, 043502 (1999).

[12] A. Lukas, B. Ovrut, and D. Waldram, hep-th/9806022, hep-th/9902071.

[13] M. Bremer, M.J. Duff, H. Lu, C.N. Pope, and K.S. Stelle, Nucl. Phys. B543, 321 (1999).

[14] S.W. Hawking and H. Reall, Phys. Rev. D 59, 023502 (1999).

[15] H. Reall, Phys. Rev. D 59, 103506 (1999). 